PROMOTING COVID-19 VACCINATION INTENTIONS

In press at Health Psychology.

This paper is not the copy of record and may not exactly replicate the final,

authoritative version of the article.

Information about Herd Immunity through Vaccination and Empathy Promote COVID-19

Vaccination Intentions

\author{
Stefan Pfattheicher ${ }^{\mathrm{a}}$, Michael Bang Petersen ${ }^{\mathrm{b}}$, and Robert Böhm ${ }^{\mathrm{c}, \mathrm{d}, \mathrm{e}}$ \\ ${ }^{\mathrm{a} D e p a r t m e n t ~ o f ~ P s y c h o l o g y ~ a n d ~ B e h a v i o u r a l ~ S c i e n c e s, ~ A a r h u s ~ U n i v e r s i t y, ~}{ }^{\mathrm{b}}$ Department of \\ Political Science, Aarhus University, ${ }^{\mathrm{c} D e p a r t m e n t}$ of Psychology, University of Copenhagen \\ ${ }^{\mathrm{d}}$ Department of Economics, University of Copenhagen; ${ }^{\mathrm{e}}$ Copenhagen Center for Social Data \\ Science (SODAS), University of Copenhagen
}

\begin{abstract}
Author Note
Stefan Pfattheicher https://orcid.org/0000-0002-0161-1570

Michael Bang Petersen https://orcid.org/0000-0002-6782-5635

Robert Böhm https://orcid.org/0000-0001-6806-0374

We have no known conflict of interest to disclose. Data, materials, and preregistrations are available at https://osf.io/4395f/

Correspondence concerning this article should be addressed to Stefan Pfattheicher, Department of Psychology and Behavioural Sciences, Aarhus University, Bartholins Allé 11, 8000, Aarhus C, Denmark. Email: sp@psy.au.dk
\end{abstract}




\begin{abstract}
Objective: An effective vaccine against COVID-19 is a desired solution to curb the spread of the disease. However, vaccine hesitancy might hinder high uptake rates and thus undermine efforts to eliminate COVID-19 once an effective vaccine became available. The present contribution addresses this issue by examining two ways of increasing the intention to get vaccinated against COVID-19.
\end{abstract}

Methods: Two pre-registered online studies were conducted $(N=2,315$ participants from the UK) in which knowledge about and beliefs in herd immunity through vaccination, as well as empathy for those most vulnerable to the virus, were either measured (Study 1) or manipulated (Study 2). As a dependent variable, individuals' self-reported vaccination intention once a vaccine against COVID-19 became available was assessed.

Results: In Study $1(N=310)$, the intention to get vaccinated against COVID-19 was correlated with knowledge about and belief in herd immunity through vaccination $(r=.58, p<.001)$, as well as with empathy for those most vulnerable to the virus $(r=.26, p<.001)$. In Study $2(N=$ $2,005)$, information about herd immunity through vaccination (Cohen's $d=0.13, p=.003$ ) and empathy (Cohen's $d=0.22, p<.001$ ) independently promoted vaccination intention.

Conclusions: The motivation to get vaccinated against COVID-19 was related to and could be causally promoted by both mere information about herd immunity through vaccination and by empathy. As such, the present research provides a better understanding of the intention to get vaccinated against COVID-19.

Keywords: COVID-19; Herd Immunity; Empathy; Prosociality; Vaccination 


\section{Information about herd immunity through vaccination and empathy promote COVID-19 vaccination intentions}

The World Health Organization describes the release of a vaccine as a "vital tool" to combatting the COVID-19 pandemic (WHO, 2020d). As this pandemic continues to severely harm societies across the globe, researchers and governments have accordingly expended tremendous effort to develop a safe and efficient vaccine against COVID-19. In fact, within only a few months of the pandemic outbreak, several vaccine candidates have successfully passed phase III of clinical testing, in which efficacy, effectiveness, and safety of the vaccine candidates were tested in large, randomized controlled trials (WHO, 2020b); moreover, as our research group finished writing the present article, an unparalleled effort proved yet ongoing — across the globe - to produce, distribute, and administer billions of doses of newly developed COVID-19 vaccines.

At the same time, WHO warns that the release of an effective vaccine will not on its own provide an end to the current pandemic (WHO, 2020d). There remains, then, a gulf between developing a vaccine and ensuring sufficient popular uptake of it (Dubé et al., 2013). To halt the spread of an infectious disease, a sufficient percentage of a population is required to become immune to it (e.g., through vaccination) such that chains of infection are interrupted-a phenomenon often referred to as "herd immunity/protection" or "community immunity/protection" (Fine et al., 2011).

Some people (including prominent politicians) have suggested to reach herd immunity against COVID-19 by intentionally exposing (low-risk) people to the virus. This approach has been rejected by the WHO and many scientists (e.g., Aschwanden, 2020; WHO, 2020a) because its consequences are scientifically problematic (e.g., long-term effects of infections are largely 
unknown) and unethical (e.g., putting people at risk of severe harm or even death). Therefore, in the present manuscript, we focus on herd immunity (community protection) through vaccination. Estimations of population-wide vaccination rates to achieve elimination of COVID-19 due to herd immunity vary widely depending on several disease- and vaccine-related parameters (Britton et al., 2020; Sanche et al., 2020), with most recent estimates reaching consensus at 60$70 \%$ as a threshold value (Clemente-Suárez et al., 2020).

In Western democracies, any mandatory vaccination policy is normatively problematic and will most likely be met with significant controversy (e.g., Sprengholz \& Betsch, 2020). From the perspective of ensuring sufficient vaccine uptake, it is therefore concerning that a significant proportion of people are hesitant to get vaccinated against COVID-19, that is, they may delay or refuse vaccination (MacDonald, 2015). Mullen O'Keefe (2020) report that in late July 2020, only $65 \%$ of US citizens are committed to getting vaccinated against COVID-19-even if a vaccine was FDA-approved and available to them at no cost. In the UK, as of mid-June 2020, only $64 \%$ of participants reported being committed to get vaccinated. Similar or even higher numbers in vaccine hesitancy can be observed in many European countries, such as Germany, Italy, and others (Lindholt et al., 2020; Murphy et al., 2020; Sherman et al., 2020), and other studies suggest that vaccine hesitancy has further increased throughout the pandemic in many countries (Ipsos Institute, 2020; Kerr et al., 2020). Additionally, there are (media) reports about people's increasing uncertainty regarding the safety of new mRNA vaccines, entering the market for the first time (Pardi et al., 2018; Siddique, 2020), which is likely to feed vaccine hesitancy even further. In summary, it could be difficult to reach uptake rates based on voluntary vaccination that would be sufficient to eliminate COVID-19.

In the case of COVID-19, this hesitancy may reflect challenges that are both 
informational and motivational. Informationally, the COVID-19 pandemic has led to an "infodemic" - that is, the deliberate and massive circulation of "wrong information to undermine the public health response" (WHO, 2020c). Given the historical abundance of conspiracy theories about vaccines, it is unsurprising that COVID-19 vaccination has also been a key target of misinformation (e.g., Imhoff \& Lamberty, 2020), something that may have led to increased vaccine hesitancy during the pandemic (Sprengholz et al., 2020).

Added to this informational challenge is an equally potent motivational challenge - that is, motivating vaccination even among those not directly at risk of severe illness from COVID19. The mortality profile of COVID-19 is strongly correlated with age (Promislow, 2020); hence, herd immunity can only be reached if large segments of society, even those not a significant risk, are willing to get vaccinated. This problem is attenuated by the fact that the supply of any efficient vaccine is exceptionally limited in the initial months after development and that vaccines in general are often less effective among the elderly due to their lower immune response (Gustafson et al., 2020). Accordingly, it is likely that some of the initial groups to be offered a vaccine include groups such as caretakers or healthcare professionals who are mainly being vaccinated not for their own sake but to protect vulnerable groups.

These challenges correspond to the determinants of vaccination decisions identified in previous research (for reviews see, Betsch et al., 2015; Brewer et al., 2018). Building on and extending previous models and theories on vaccination behavior and health behavior change more generally (e.g., 5A taxonomy, Thomson et al., 2016; 3C model, MacDonald, 2015; Health Belief Model, Abraham \& Sheeran, 2007), one of the most extensive theoretical models in this domain is the $5 \mathrm{C}$ model (Betsch et al., 2018). It suggests that vaccination becomes more likely with people's confidence (i.e., trust in the effectiveness and safety of vaccines and trust in 
policymakers who recommend them and the system that delivers them) and collective responsibility (i.e., knowledge and willingness to protect others by one's own vaccination by means of herd immunity). In contrast, vaccination should become less likely with complacency (i.e., low perceived risks of diseases), constraints (i.e., structural or psychological barriers, e.g., access, lack of self-control), and calculation (i.e., engagement in extensive information searching). Although previous research has shown that each of these factors is related to people's vaccination intentions and behaviors (Betsch et al., 2018, 2019), the focus of the present contribution is on the informational and motivational challenge with regard to people's collective responsibility (i.e., protecting others), as this appears of particular relevance to COVID-19 due to its heterogeneous risk profile.

\section{Providing information about herd immunity through vaccination: Vaccination as a prosocial act}

Vaccination provides a direct (personal) benefit by eliminating or at least reducing the likelihood of infection with the particular disease. In addition, most vaccines also provide indirect benefits to other, unvaccinated people by contributing to herd immunity. Considering the costs associated with getting vaccinated (e.g., time of getting vaccinated, risk of vaccine-adverse events), vaccination constitutes a prosocial act, particularly when there is already high vaccine uptake in the population or when people are themselves not at risk of severe disease symptoms (e.g., Betsch et al., 2013; Böhm et al., 2016). ${ }^{1}$ The latter is of particular importance in the context of the COVID-19 pandemic.

Research conducted prior to the COVID-19 pandemic has shown that a considerable

\footnotetext{
${ }^{1}$ Note that with increasing vaccine uptake in the population, the likelihood of contracting the disease oneself decreases because of the herd immunity benefits provided by others. As such, free-riding incentives of benefitting from rather than contributing to herd immunity increase (for a formal description, see e.g., Bauch \& Earn, 2004; Böhm et al., 2016).
} 
number of people does not know about herd immunity through vaccination (about one third of the US participants sampled in Griffith et al., 2020, and Logan et al., 2018) and that vaccination intention increases when informing people about herd immunity (for a review, see Hakim et al., 2019). For example, in a cross-cultural online experiment, Betsch and colleagues (2017; see also Betsch \& Böhm, 2018; Sprengholz \& Betsch, 2020) did versus did not provide information about herd immunity (either with a text-based explanation or an interactive simulation) and found that participants increased their vaccination intention, particularly in Western countries and when there were low individual incentives to get vaccinated (i.e., an already high vaccination rate). Furthermore, it has been shown that vaccination and, thus, contributing to herd immunity, is represented as a "social contract," indicated by vaccinators' tendency to "reward" vaccinated others but to "punish" unvaccinated others (Korn et al., 2020). In sum, knowledge about herd immunity through vaccination could increase people's intention to get vaccinated against COVID-19.

\section{Increasing the motivation to contribute to herd immunity through vaccination: Empathy, prosociality, and health behaviors}

Understanding the prosocial nature of vaccination is one thing; being motivated to engage in this prosocial act is another. In understanding the latter decision, theoretical and empirical work is increasingly oriented towards the concept of empathy. Empathy can be defined as an umbrella term capturing the range of a person's responses to another individual's experience (Hodges \& Myers, 2007). Past research has convincingly demonstrated the beneficial consequences of both affective as well as cognitive empathy for the welfare of others. Specifically, cognitive empathy (i.e., taking the perspective of others) has been linked to reductions of inter-group conflicts and prejudice, while affective empathy (i.e., a concern for and 
an understanding of vulnerable others) has been shown to promote altruism and caring (Batson, 2011; Sassenrath et al., 2016; Todd \& Burgmer, 2013).

In the modern health context, affective empathy in particular has been shown to improve health outcomes. For instance, physicians' affective empathy levels have been positively associated with reduced metabolic complications and better self-care in diabetic patients (Hojat et al., 2011; Del Canale et al., 2012). Affective empathy has further been shown to promote healthcare professionals' adherence to handwashing compliance in order to protect others in hospitals (Sassenrath et al., 2016; Grant \& Hofmann, 2011). In the context of COVID-19, it has further been shown that affective empathy promotes the motivation to engage in physical distancing and in wearing of face masks (Pfattheicher et al., 2020).

Overall, past research suggests that empathy motivates a variety of behavioral outcomes aimed at helping and protecting vulnerable others. Based on the notion that getting vaccinated is a prosocial act that helps to protect vulnerable others, we argue that empathy for those most vulnerable to the virus can promote the motivation to get vaccinated against COVID-19. Indirect evidence for this hypothesis comes from Böhm et al. (2019), who have shown that the motivation to get vaccinated increases when other people are themselves unable to get vaccinated (e.g., due to a chronic disease); it might be that empathy for those vulnerable others has driven individuals' increased vaccination motivation. In sum, increasing people's empathy for those most vulnerable to COVID-19 could increase their intention to get vaccinated against COVID-19.

\section{The present research}

The present contribution seeks to address both the informational and motivational challenges related to motivating voluntary uptake of a COVID-19 vaccine. To meet the 
informational challenge, we specifically tested whether information that establishes accurate public perceptions of the concept of herd immunity through vaccination will increase people's vaccination intention. To meet the motivational challenge, we further tested whether inductions of empathy for those most vulnerable to COVID-19 can motivate people to get vaccinated in order to protect vulnerable others, even if people are themselves not at a significant risk. Taken together, we empirically tested the hypotheses that (i) increased knowledge about and belief in herd immunity through vaccination as well as (ii) stronger empathic concern for those most vulnerable to the virus are associated with the intention to get vaccinated against COVID-19 (Study 1) and can (causally) promote this intention (Study 2). Finally, we also tested the interaction between mere information and empathy, that is, whether combining herd immunity information with the "emotional fire" of affective empathy (Batson et al., 1995, p. 1053) has a synergetic effect on the intention to get vaccinated against COVID-19.

Our research contributes to both theory and practice. From a theoretical perspective and in line with recent research (e.g., Böhm et al., 2019, 2016; Korn et al., 2020; Shim et al., 2012), the present research conceptualizes getting vaccinated as a prosocial act: Through vaccination, one can contribute to protecting those at high risk from a COVID-19 infection and/or who cannot get vaccinated, like those suffering from certain chronic diseases (Rubin et al., 2014). We show

that prosocial vaccination may be promoted by correct perceptions about the prosocial aspects of vaccination decisions as well as by emotions related to others' potential suffering. In other words, prosocial vaccination appears to be related to both informational (or cognitive) and motivational (or affective) aspects of human prosociality.

\section{Overall Methods}

The two studies reported below were conducted in line with the Declaration of Helsinki 
and the guidelines of the American Psychological Association (APA). Institutional review boards or committees are not mandatory for survey-based research in the country where the investigator responsible for the present studies (first author) is employed (Denmark; see section 14(2) of the Committee Act for National Committee of Health Research Ethics or https://en.nvk.dk/how-tonotify/what-to-notify). Participants gave informed consent before starting the study. There was no deception of participants. Both studies were preregistered. Data, materials, and the preregistrations are available on the Open Science Framework (OSF; https://osf.io/4395f/).

All results remain robust in terms of significance levels and effect sizes when controlling for participants' gender, age, and household size. Furthermore, none of these demographic variables significantly moderated the relationships among herd immunity, empathy and the intention to get vaccinated in either studies ( $p$-values of all tested interactions were $>.05$ ). We applied attention checks to secure high data quality ("This is an attention check. Please answer: Strongly agree."); in each study, less than $5.7 \%$ of participants failed the attention checks and were excluded from all analyses (as preregistered). All results hold when all subjects are included.

\section{Study 1}

\section{Method}

Study 1 consists of a sample from the UK which was collected on September 7, 2020. At that time, several vaccine candidates were in clinical trial phase III, but none of the involved companies or public institutions had applied for official approval from national health authorities (this status also holds for Study 2). At the time the two studies were conducted (in week 36 and on Monday in week 37, i.e., early September 2020), there were 12,685 confirmed new cases and 347,152 cumulative cases in total in the UK (numbers refer to the entire week 36); there were 52 
new deaths and 41,551 deaths in total ascribed to COVID-19 in the UK (European Centre for Disease Prevention and Control, 2020).

\section{Participants}

Participants were recruited via the crowdsourcing platform Prolific Academic to complete a short survey on "coronavirus (COVID-19)" in exchange for $£ 1.13(\sim \$ 1.46)$. Sample size was based on an a priori power analysis to detect effects of $r>.18$ with high statistical power (Power $=.90$; alpha-level of .05, two-tailed). Based on this analysis, we aimed to collect at least 300 participants (final samples: 310 participants, $68.4 \%$ female, $M_{\text {age }}=34.64, S D=$ 13.33, household size $M=2.95, S D=1.43$ ).

\section{Measures}

Herd immunity through vaccination. We measured knowledge about and belief in herd immunity by two items. First, participants read, "How likely is this statement to be true? 'When you get vaccinated against the coronavirus, you're protecting not only yourself but also your community: When a sufficient percentage of a population has become immune to an infection (e.g., through vaccination), this reduces the likelihood of infection for individuals who lack immunity."” Labels ranged from 1 (very unlikely to be true) to 5 (very likely to be true). Regarding the second item, participants read on the next page, "On the last page, we referred to the concept of herd immunity: 'When a sufficient percentage [...] who lack immunity.' How much do you agree with the following statement: Herd immunity through vaccination will be an important measure to slow down the spread of the coronavirus." Labels ranged from 1 (strongly disagree) to 5 (strongly agree). The two items were highly correlated $(r=.70, p<.001)$ and averaged as preregistered $(M=4.25, S D=0.94)$.

Empathy. We measured affective empathy for those most vulnerable to the virus with 
the three items from Pfattheicher et al. (2020). The items (Cronbach's $\alpha=.89$ ) read, "I am very concerned about ... / I feel compassion for ... / I am quite moved by what can happen to ... those most vulnerable to coronavirus (COVID-19)." Labels ranged from 1 (strongly disagree) to 5 (strongly agree). Relatively high levels of empathy were observed $(M=4.17, S D=0.73)$.

Vaccination intention. In line with previous research (Betsch et al., 2013, 2017; Betsch \& Böhm, 2018), we measured the intention to get vaccinated against COVID-19 with one simple and straightforward item: "If you had the opportunity to get vaccinated against the coronavirus (COVID-19) immediately, what would you do?’ Labels ranged from 1 (I would definitely not get vaccinated) to 5 (I would definitely get vaccinated). We observed medium to high levels of vaccination intention with considerable variability $(M=3.96, S D=1.37)$.

Personality. To apply a conservative test to the correlations among herd immunity through vaccination, empathy, and vaccination intention, we aimed at showing the robustness of effects beyond basic personality traits (as preregistered). In fact, personality traits typically already capture a significant amount of variance in individual differences in motivations and behavior (e.g., Soto, 2019; Thielmann et al., 2020), that said, in our analyses, we controlled for basic personality traits by including the prominent HEXACO personality dimensions as covariates. The HEXACO personality model (Honesty-Humility, Emotionality, Extraversion, Agreeableness, Conscientiousness, and Openness) was assessed at the beginning of the study with the 60-item version of the HEXACO Personality Inventory-Revised (HEXACO-60; Ashton \& Lee, 2009; 10 items per dimension). All dimensions had good reliability (all $\alpha \mathrm{s}>.77$ ). None of the HEXACO dimensions played a major role regarding vaccination intention; the largest correlation was found for Emotionality, Pearson's $r=.14, p=.013$ (see the OSF for detailed results). 


\section{Results and discussion}

In line with the idea that knowing about and believing in herd immunity through vaccination relates positively to the intention to get vaccinated, results revealed a positive correlation between herd immunity knowledge/belief and the intention to get vaccinated against COVID-19, Pearson's $r=.58, p<.001$. The correlation holds when the HEXACO personality dimensions were controlled for, $r_{\text {partial }}=.55, p<.001$ (see OSF for detailed results). In a similar vein, empathy for those most vulnerable to the virus relates positively to vaccination intention, $r$ $=.26, p<.001$. Again, the correlation holds when the HEXACO personality dimensions were controlled for, $r_{\text {partial }}=.19, p<.001$ (see OSF for detailed results). When herd immunity through vaccination and empathy were simultaneously entered in an OLS linear regression model with vaccination intention as the dependent variable (non-preregistered), herd immunity was still significant, $B=0.81, S E=0.07, p<.001$, whereas the effect of empathy decreased and was no longer significant at conventional significance levels, $B=0.16, S E=0.09, p=.073$ (including or excluding the HEXACO did not play a role in this analysis).

In sum, and in support of the preregistered hypotheses, the first study shows that the intention to get vaccinated against COVID-19 relates to knowledge about and belief in herd immunity through vaccination, as well as to empathy for those most vulnerable to the virus. In a second study, we went one step further and sought to test whether one can use information about herd immunity through vaccination and empathy to promote (in a causal sense) the motivation to get vaccinated against COVID-19.

\section{Study 2}

\section{Method}

\section{Participants}


Participants were recruited via the crowdsourcing platform Prolific Academic to complete a short survey on "coronavirus (COVID-19)" in exchange for $£ 0.50(\sim \$ 0.65)$. We aimed to collect 2,000 participants for Study 2 (four between-subjects conditions). With this sample size, we can detect main effects and interaction effects of Cohen's $f>0.062$ with sufficient statistical power (Power $=.80$; alpha-level of .05 , two-tailed). In addition, with $n=500$ in each condition, we obtain statistical power of $>.97$ to show effects larger than Cohen's $d>.25$ between two conditions ( simple effects). Study 2 (final $N=2,005 ; 63.4 \%$ female, $M_{\text {age }}=34.34$, $S D=13.42$, household size $M=3.10, S D=1.38$ ) was run in the UK on September 5, 2020. Participants were recruited as in Study 1; we made certain that participants were unable to participate in both studies reported in the present article.

\section{Experimental conditions}

Participants were randomly assigned to one of four conditions: the "herd immunity condition" $(n=498)$, the "empathy condition" $(n=495)$, the "empathy + herd immunity condition" ( $n=519)$, and the "control condition" $(n=493)$. Full instruction materials of the conditions can be found on the OSF (https://osf.io/4395f/).

Participants in the herd immunity condition were informed about the concept of herd immunity through vaccination and its effects, in line with previous research (Betsch et al. 2017; Betsch \& Böhm, 2018). Participants read, "Did you know that when you get vaccinated, you're protecting yourself and your community? This concept is called herd immunity. Herd immunity denotes the effect that occurs when acquired immunity against a pathogen within a population (the "herd"), generated through infection or vaccination, has reached such a level that nonimmune individuals in the population are also protected because the pathogen can no longer be transmitted. Thus, if you get vaccinated, then you can protect others who are not vaccinated." On 
the page where the text about herd immunity through vaccination was presented, the "next" button to proceed with the study was displayed only after 15 seconds had elapsed.

Empathy was induced following the basic procedure of Pfattheicher et al. (2020). That is, participants in the empathy condition read an empathy-promoting short story (taken from The Guardian) about two siblings who shared a close bond and worked together in a nursing facility that specialized in the care of elderly people with cognitive issues. The story focused on one of the siblings, Josh, who was described as a "helpful, empathetic nurse who makes things easier for everybody." Participants read further, "Both [siblings] got tested for COVID-19 on the same day in June. Both tests came back positive. Yet only one of them survived.” Participants learned that it was Josh who died. On the page where the text was presented, the "next" button to proceed with the study was displayed only after 60 seconds had elapsed.

Participants in the empathy + herd immunity condition first read the empathy eliciting text about the two siblings, then they read the information about herd immunity through vaccination. In the control condition, no text and information were given, resembling a situation of no intervention. In order to avoid unequal dropout between conditions, participants in all conditions read all texts: Participants in the empathy condition read the text about herd immunity at the end of the study (before the concluding demographics were assessed); participants in the herd immunity condition instead read the empathy eliciting text at the end of the study, while participants in the control condition read both texts at the end of the study.

\section{Measures}

Vaccination intention. The motivation to get vaccinated against COVID-19 was measured with the same items as in Study 1. Labels ranged from 1 (I would definitely not get vaccinated) to 7 (I would definitely get vaccinated). 
State empathy. After the item measuring vaccination intention, participants responded to three items assessing state empathy, as in previous research (Pfattheicher et al., 2019, 2020). The items $(\alpha=.87)$ read, "Right now, I feel compassion for those most vulnerable to coronavirus (COVID-19)"; "Right now, I am touched by those most vulnerable to coronavirus (COVID-19)"; and "Right now, I am quite moved by what can happen to those most vulnerable to coronavirus (COVID-19).” Labels ranged from 1 (strongly disagree) to 7 (strongly agree).

Additional measures. To test the possibility that information about herd immunity through vaccination and an additional empathy-induction makes one sensitive for one's own vulnerability, which in turn promotes one's (egoistic) motivation to get vaccinated, we assessed individuals' subjective vulnerability. We tested whether subjectively considering themselves as vulnerable (versus less so) would lead to increased vaccination intention (to protect themselves) after reading about herd immunity through vaccination and the empathy-induction. In line with Pfattheicher et al. (2020), participants' subjective own vulnerability was measured with one item ("What do you think the danger is that you will be infected with the novel coronavirus?"). We also measured perceived vulnerability of others with one item ("What do you think the danger is that other people will be infected with the novel coronavirus?"). Labels ranged from 1 (very harmless) to 7 (very dangerous). We also assessed individuals' objective vulnerability—whether a participant is in a high-risk group $(n=551,36.1 \%)$ or not $(n=973,63.8 \%)$, according to the criteria of the German federal government agency for disease control and prevention, the Robert Koch Institute (e.g., whether one is above 60 years old, a smoker, chronically ill).

\section{Results and discussion}

We successfully induced empathy, in that state empathy was increased in the empathy 
condition (vs. control condition, $p<.001$, Cohen's $d=0.22$ ) but not in the herd immunity condition (vs. control condition, $p=.76$, Cohen's $d=0.01$; for details see Supplementary Material at https://osf.io/4395f/).

Next, we tested the key (preregistered) hypotheses regarding whether providing mere information about herd immunity through vaccination, and whether inducing empathy, increase the intention to get vaccinated against COVID-19. Means of the conditions are presented in Figure 1. Analysis of variance yielded a significant main effect of the herd immunity information, $F(1,2001)=8.94 p=.003, \eta^{2}=.004$, in that participants report higher vaccination intention when the informative text about herd immunity through vaccination was provided $(M=$ $5.65, S D=1.77)$ compared to when this was not the case $(M=5.41, S D=1.88$; Cohen's $d=$ 0.13). We can also document a significant main effect of the experimental empathy-induction, $F(1,2001)=13.70, p<.001, \eta^{2}=.01$, in that participants report higher vaccination intention when the empathy-eliciting text was provided $(M=5.68, S D=1.71)$ compared to when this was not the case $(M=5.38, S D=1.93$; Cohen's $d=0.22)$. We did not find a significant interaction between herd immunity and empathy, $F(1,2001)=0.97, p=.33, \eta^{2}<.01$. These results indicate that herd immunity and empathy have independent and additive effects on the motivation to get vaccinated. We discuss the implications of these important findings in the discussion section below.

To facilitate comprehension of the effect of the experimental inductions, it is relevant to note that the percentage of participants who scored below the midpoint on the item measuring vaccination intentions decreased from $18.7 \%$ in the control condition to $16.1 \%$ in the herd immunity condition, to $12.9 \%$ in the empathy condition, and finally to $10.6 \%$ in the herd immunity + empathy condition. In contrast, the percentage of participants who scored above the 


\section{Figure 1}

Vaccination intention (1-7) depending on the experimental conditions (Study 2)

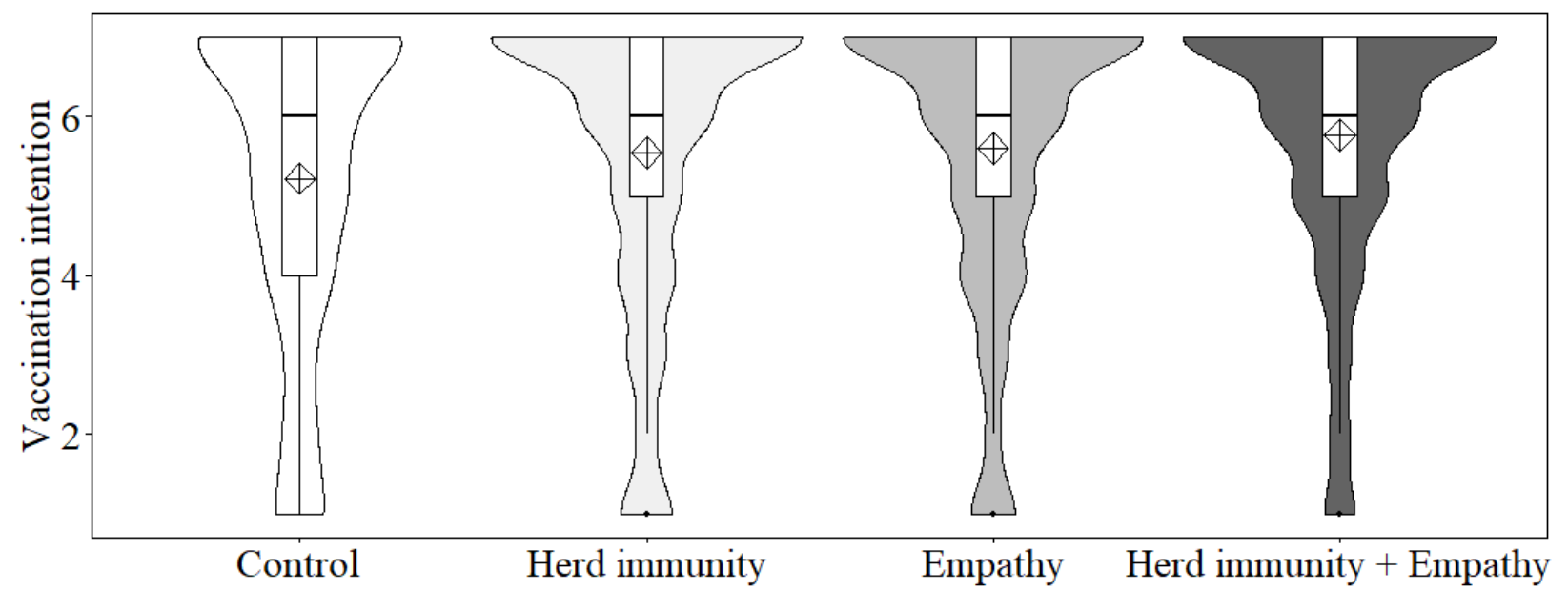

Note. The vertical box indicates the interquartile range from the 25 th to the 75 th percentile, including the median (line) and the mean value (diamond); the shaded areas indicate the density of observations. 
midpoint on the item measuring vaccination intentions increased from $70.4 \%$ in the control condition to $75.5 \%$ in the herd immunity condition, to $77.4 \%$ in the empathy condition, and finally to $82.7 \%$ in the herd immunity + empathy condition. This pattern suggests that the manipulations not only had some effects on those who had no basic motivation to get vaccinated but also further increased the motivation in those who already have a relatively high basic motivation.

Further analyses (reported in detail in the Supplementary Material available at https://osf.io/4395f/) showed that (i) higher levels of state empathy are directly related to participants' vaccination intention $(r=.23, p<.001)$, (ii) state empathy mediates the effect of the experimental empathy-induction (but not the effect of the information about herd immunity through vaccination) on the intention to get vaccinated, (iii) the experimental empathy-induction slightly increased participants' subjective own vulnerability $(\eta 2=.004, p=.006)$ but not the perception of others' vulnerability $(\eta 2=.00, p=.091)$, (iv) subjective vulnerability was positively correlated with vaccination intention (self: $r=.25, p<.001$; other: $r=.32, p<.001$ ), and (v) those being objectively more vulnerable (being in the risk group) had a higher vaccination intention than those not in the risk group (Cohen's $d=0.14, p=.022$ ). In addition, none of the vulnerability measures (objective and subjective vulnerability) significantly moderated the effect of empathy and herd immunity on the intention to get vaccinated.

\section{General discussion}

The present studies provide four major findings. First, with increasing knowledge about and beliefs in herd immunity through vaccination, the intention to vaccinate against COVID-19 increased. Second, providing information about herd immunity through vaccination increased vaccination intention. Third, empathy for those most vulnerable to the virus also related to the 
intention to get vaccinated against COVID-19. Fourth, inducing empathy increased people's vaccination intention.

The findings of the present studies can be considered from multiple perspectives. First, the finding that information about herd immunity through vaccination and empathy both promote the intention to get vaccinated against COVID-19 is noteworthy given that a significant number of participants either have a very low intention to get vaccinated against COVID-19 or a very strong intention to do so-and who are thus less likely to be influenced by such interventions. Even though these strong motivations exist in the context of a COVID-19 vaccine, and in the context of vaccines in general (de Figueiredo et al., 2020), information about herd immunity through vaccination and empathy both were nonetheless able to alter (i.e., to promote) vaccination intention. In this regard, the present work contributes to the existing literature by showing that providing information about both herd immunity through vaccination and empathy can increase vaccination intention even when low or very high motivation exists in a significant number of people.

Second, when changing intentions (and attitudes) in an emotionally loaded and highstakes context — such as whether or not one should get vaccinated during a pandemic — reactance is always a risk. Reactance occurs when people believe that their freedom or control is threatened or reduced (Brehm, 1966; Rosenberg \& Siegel, 2018). Advising people what to do (via information about herd immunity through vaccination) or "manipulating" their emotional state (such as empathy) could potentially alter people's perception of their own freedom or control, bearing the risk of reactance - and ultimately the reduced intention to get vaccinated. For instance, such psychological reactance has been shown to occur when mandating vaccination (Sprengholz \& Betsch, 2020). Even though reactance was a risk in our studies, the present work 
shows that information about herd immunity through vaccination and empathy can nonetheless increase overall intention to get vaccinated.

Third, the present work relates to previous work that has conceptualized vaccination as a prosocial act — based on the notion that most vaccines also provide indirect benefits to other, unvaccinated people by contributing to herd immunity (Bauch \& Earn, 2004; Betsch et al., 2013; Böhm et al, 2016; Chapman et al., 2012). In line with this conceptualization, previous research has shown that people's collective responsibility (i.e., protecting others through one's own vaccination) is one important motivation for the decision to get vaccinated (Betsch et al., 2018). In this regard, the present research shows two ways of how to increase collective responsibility: by informing people about herd immunity through vaccination, and by promoting empathy.

Fourth, the findings are also noteworthy from the perspective of research on empathy. The present work (i) extends classic work on empathy and shows that empathy also reveals its prosocial impact in a high-stakes situation of getting vaccinated during a pandemic; (ii) shows that empathy can work in conjunction with providing mere information, in that emotion and information supporting (rather than inhibiting) each other's positive effects on vaccination intention; and (iii) offers one of the largest preregistered experiments testing the effect of empathy on prosocial tendencies (cf. the meta-analysis by McAuliffe et al., 2020).

The present research could have policy implications once a vaccine is widely available to the general public. Specifically, in the effort to reach high numbers of people getting vaccinated against COVID-19 once a vaccination is available, the present research suggests that a combination of informational content and emotional content might be a possible way to increase the motivation to get vaccinated. This is particularly important in light of the significant proportion of people who are hesitant to get vaccinated against COVID-19 (Betsch, Korn, et al., 
2020; Lindholt et al., 2020; Mullen O’Keefe, 2020). In fact, two independent, additive main effects of herd immunity through vaccination and empathy were found, and accordingly, the strongest motivation to get vaccinated was found in the condition where both the informational content (herd immunity through vaccination) and the emotional content (empathic text) were provided (see Figure 1). As such, when designing interventions and communication materials to promote the motivation to get vaccinated against COVID-19, policymakers could consider combining informational content with emotional content. Such intervention, however, must be again evaluated empirically.

\section{Limitations}

In discussing the findings, we do want to acknowledge limitations of the present research and point to future directions. First, while conducting the studies and writing the article, it was yet unclear whether and to what degree the new COVID-19 vaccines would provide herd immunity (e.g., whether vaccinated people can still transmit the virus). Our manipulations and findings build on the assumption of vaccination serve a prosocial act by contributing to herd immunity, but the effects could differ quantitatively and qualitatively if herd immunity effects are small or apply only to specific conditions. In any case, our results suggest that vaccines with prosocial (herd immunity/community protection) benefits have the potential to motivate vaccination more than vaccines without such indirect benefits.

Second, we want to acknowledge that the observed effect sizes in the experimental study were small. We argue, however, that small effect sizes matter in the context of a pandemic (cf. Funder \& Ozer, 2019). However, it remains an open question what the "true" effect sizes are in real life contexts. Relatedly, we do not claim or show that increasing collective responsibility is the only or possibly even the strongest intervention for increasing vaccination intentions. In fact, 
previous research has documented the importance of other factors, such as confidence in the safety and effectiveness of vaccines and trust in health authorities who develop and license vaccines (e.g., Betsch et al., 2018; Geiger et al., in press). Furthermore, concerns for collective responsibility might differ between different vaccines and populations (both absolutely and relatively to other factors). Therefore, more research is needed to show whether interventions aimed at increasing prosocial vaccinations constitute a promising approach to increasing vaccine uptake.

Third, we did not assess additional potential moderators, such as whether a person ever tested positive (which was below $1 \%$ of the UK population at the time the study was conducted); people's general (prior) beliefs and skepticism about vaccines (as e.g., conceptualized in the 5C psychological antecedents of vaccination model; Betsch et al., 2018); concerns about the short development period of the vaccines against COVID-19 (as well as other factors such as the sample size of the phase III of clinical testing); or whether people live in an area with high infection rates. Regarding the latter factor, recent research shows that concerns for others (which is related to empathy) increases the intention to get vaccinated against COVID-19 more in sparsely rather than densely populated areas (Jung \& Albarracín, 2020). In sum, we want to acknowledge that the present work remains silent regarding additional moderators likely relevant during the COVID-19 pandemic.

Fourth, we further want to highlight that the present studies were conducted at a time the vaccines against COVID-19 were in the process of development (some of them in clinical trial phase III) but were not yet approved. As such, the intention to get vaccinated might not correspond to actual behavior at the time the vaccines were approved, or the time people were able to become vaccinated. Although intentions may not perfectly match behavior, it was the best 
proxy available in a situation where a vaccine does not yet exist (at the time the studies were conducted). In this regard, the results of the present work apply to the situation before the vaccine against COVID-19 was approved, and when there was not yet a broad discussion about vaccines, their rapid development, and their safety profile in the media. As such, it remains an open question whether improving (or correcting) beliefs about herd immunity through vaccination and empathy are able to motivate people to get vaccinated when vaccines were actually available.

Finally, we want to acknowledge that the two studies were conducted in a Western country only (the UK), and that the samples were not representative of the entire population in this country (also with respect to vaccination intentions); the studies were conducted online using self-reports; and the intervention (providing information about herd immunity through vaccination and inducing empathy) was applied and tested on only a short-term scale. As such, it might be useful to replicate the present studies' findings using different stimuli as well as representative samples; to conduct the studies in other (non-)Western countries; and to measure the long-term consequences of providing people with information, especially when attitudes about vaccines become more chronic due to repeated exposure to (mis)information.

\section{Conclusion}

We argue that these shortcomings do not limit the basic conclusions: The motivation to get vaccinated against COVID-19 was related to and could be causally promoted by both mere information about herd immunity through vaccination and by empathy. As such, the present research provides a better understanding of the intention to get vaccinated against COVID-19. 


\section{References}

Abraham, C., \& Sheeran, P. (2007). The health belief model. In S. Ayers, A. Baum, C. McManus, S. Newman, K. Wallston, J. Weinman, et al. (Eds.), Cambridge Handbook of Psychology, Health and Medicine (pp. 97-102). Cambridge University Press. https://doi.org/10.1017/CBO9780511543579.022

Aschwanden C. (2020). The false promise of herd immunity for COVID-19. Nature, 587(7832), 26-28. https://doi.org/10.1038/d41586-020-02948-4

Ashton, M. C. \& Lee, K. (2009). The HEXACO-60: A short measure of the major dimensions of personality. Journal of Personality Assessment, 91(4), 340-345. https://doi.org/10.1080/00223890902935878

Batson, C. D (2011). Altruism in humans. Oxford, UK: Oxford UniversityPress.

Batson, C. D., Klein, T. R., Highberger, L., \& Shaw, L. L. (1995). Immorality from empathyinduced altruism: When compassion and justice conflict. Journal of Personality and Social Psychology, 68(6), 1042-1054. https://doi.org/10.1037/0022-3514.68.6.1042

Bauch, C. T., \& Earn, D. J. D. (2004). Vaccination and the theory of games. Proceedings of the National Academy of Sciences of the United States of America, 101(36), 13391-13394. https://doi.org/10.1073/pnas.0403823101

Betsch, C., \& Böhm, R. (2018). Moral values do not affect prosocial vaccination. Nature Human Behaviour, 2(12), 881-882. https://doi.org/10.1038/s41562-018-0478-1

Betsch, C., Böhm, R., \& Chapman, G. B. (2015). Using Behavioral Insights to Increase Vaccination Policy Effectiveness. Policy Insights from the Behavioral and Brain Sciences, 2(1), 61-73. https://doi.org/10.1177/2372732215600716

Betsch, C., Böhm, R., \& Korn, L. (2013). Inviting free-riders or appealing to prosocial behavior? Game-theoretical reflections on communicating herd immunity in vaccine advocacy. Health Psychology, 32(9), 978-985. https://doi.org/10.1037/a0031590 
Betsch, C., Böhm, R., Korn, L., \& Holtmann, C. (2017). On the benefits of explaining herd immunity in vaccine advocacy. Nature Human Behaviour, 1, Article 0056. https://doi.org/10.1038/s41562-017-0056

Betsch, C., Schmid, P., Heinemeier, D., Korn, L., Holtmann, C., \& Böhm, R. (2018). Beyond confidence: Development of a measure assessing the 5C psychological antecedents of vaccination. PloS ONE, 13(12), Article e0208601. https://doi.org/10.1371/journal.pone.0208601

Böhm, R., Betsch, C., \& Korn, L. (2016). Selfish-rational nonvaccination: Experimental evidence from an interactive vaccination game. Journal of Economic Behavior \& Organization, 131, 183-195. https://doi.org/10.1016/j.jebo.2015.11.008

Böhm, R., Meier, N. W., Groß, M., Korn, L., \& Betsch, C. (2019). The willingness to vaccinate increases when vaccination protects others who have low responsibility for not being vaccinated. Journal of Behavioral Medicine, 42(3), 381-391. https://doi.org/10.1007/s10865-018-9985-9

Brehm, J. W. (1966). A theory of psychological reactance. Oxford, England: Academic Press. Brewer, N. T., Chapman, G. B., Rothman, A. J., Leask, J., \& Kempe, A. (2017). Increasing Vaccination: Putting Psychological Science Into Action. Psychological Science in the Public Interest, 18(3), 149-207. https://doi.org/10.1177/1529100618760521

Britton, T., Ball, F., \& Trapman, P. (2020). A mathematical model reveals the influence of population heterogeneity on herd immunity to SARS-CoV-2. Science, 369(6505), 846849. https://doi.org/10.1126/science.abc6810

Chapman, G. B., Li, M., Vietri, J., Ibuka, Y., Thomas, D., Yoon, H., \& Galvani, A. P. (2012). Using game theory to examine incentives in influenza vaccination behavior. Psychological Science, 23(9), 1008-1015. https://doi.org/10.1177/0956797612437606 Clemente-Suárez, V. J., Hormeño-Holgado, A., Jiménez, M., Benitez-Agudelo, J. C., NavarroJiménez, E., Perez-Palencia, N., Maestre-Serrano, R., Laborde-Cárdenas, C. C., \& Tornero- 
Aguilera, J. F. (2020). Dynamics of population immunity due to the herd effect in the COVID-19 pandemic. Vaccines, 8(2), 236. https://doi.org/10.3390/vaccines8020236 de Figueiredo, A., Simas, C., Karafillakis, E., Paterson, P., \& Larson, H. J. (2020). Mapping global trends in vaccine confidence and investigating barriers to vaccine uptake: A largescale retrospective temporal modelling study. The Lancet, 396(10255), 898-908. https://doi.org/10.1016/S0140-6736(20)31558-0

Del Canale, S., Louis, D. Z., Maio, V., Wang, X., Rossi, G., Hojat, M., \& Gonnella, J. S. (2012). The relationship between physician empathy and disease complications: An empirical study of primary care physicians and their diabetic patients in Parma, Italy. Academic Medicine, 87(9), 1243-1249. https://doi.org/10.1097/ACM.0b013e3182628fbf

Dubé, E., Laberge, C., Guay, M., Bramadat, P., Roy, R., \& Bettinger, J. A. (2013). Vaccine hesitancy: An overview. Human Vaccines \& Immunotherapeutics, 9(8), 1763-1773. https://doi.org/10.4161/hv.24657

European Centre for Disease Prevention and Control. (2020). Data on 14-day notification rate of new COVID-19 cases and deaths. ECDC. https://www.ecdc.europa.eu/en/publications$\underline{\text { data/data-national-14-day-notification-rate-covid-19 }}$

Fine, P., Eames, K., \& Heymann, D. L. (2011). "Herd immunity": A rough guide. Clinical Infectious Diseases, 52(7), 911-916. https://doi.org/10.1093/cid/cir007

Funder, D. C., \& Ozer, D. J. (2019). Evaluating effect size in psychological research: Sense and nonsense. Advances in Methods and Practices in Psychological Science, 2(2), 156-168. https://doi.org/10.1177/2515245919847202

Geiger, M., Rees, F., Lilleholt, L., Santana, A. P., Zettler, I., Wilhelm, O., Betsch, C., \& Böhm, R. (in press). The 7Cs of vaccination readiness. European Journal of Psychological Assessment. 
Grant, A. M., \& Hofmann, D. A. (2011). It's not all about me: Motivating hand hygiene among health care professionals by focusing on patients. Psychological Science, 22(12), 14941499. https://doi.org/10.1177/0956797611419172

Griffith, B. C., Ulrich, A. K., Becker, A. B., Nederhoff, D., Koch, B., Awan, F. A., \& Basta, N. E. (2020). Does education about local vaccination rates and the importance of herd immunity change US parents' concern about measles? Vaccine, 38(50), 8040-8048. https://doi.org/10.1016/j.vaccine.2020.09.076

Gustafson, C. E., Kim, C., Weyand, C. M., \& Goronzy, J. J. (2020). Influence of immune aging on vaccine responses. Journal of Allergy and Clinical Immunology, 145, 1309-1321. https://doi.org/10.1016/j.jaci.2020.03.017

Hakim, H., Provencher, T., Chambers, C. T., Driedger, S. M., Dube, E., Gavaruzzi, T., Giguere, A., Ivers, N. M., MacDonald, S., Paquette, J. S., Wilson, K., Reinharz, D., \& Witteman, H. O. (2019). Interventions to help people understand community immunity: A systematic review. Vaccine, 37(2), 235-247. https://doi.org/10.1016/j.vaccine.2018.11.016

Hayes, A. F. (2018). Introduction to mediation, moderation, and conditional process analysis: A regression-based approach (2nd ed.). Guilford Press.

Hodges, S. D., \& Myers, M. W. (2007). Empathy. In R. F. Baumeister, \& K. D. Vohs (Eds.), Encyclopedia of Social Psychology (pp. 297-298). Sage. https://www.doi.org/10.4135/9781412956253.n179

Hojat, M., Louis, D. Z., Markham, F. W., Wender, R., Rabinowitz, C., \& Gonnella, J. S. (2011). Physicians' empathy and clinical outcomes for diabetic patients. Academic Medicine, 86(3), 359-364. https://doi.org/10.1097/acm.0b013e3182086fe1

Imhoff, R., \& Lamberty, P. (2020). A bioweapon or a hoax? The link between distinct conspiracy beliefs about the coronavirus disease (COVID-19) outbreak and pandemic behavior. Social Psychological and Personality Science, 11(8), 1110-1118. https://doi.org/10.1177/1948550620934692 
Ipsos. (2020). COVID-19 vaccination intent is decreasing globally. Ipsos. https://www.ipsos.com/en/global-attitudes-covid-19-vaccine-october-2020

Jung, H., \& Albarracín, D. (2021). Concerns for others increases the likelihood of vaccination against influenza and COVID-19 more in sparsely rather than densely populated areas. Proceedings of the National Academy of Sciences, 118(1).

https://doi.org/10.1073/pnas.2007538118

Kerr, J. R., Schneider, C. R., Recchia, G., Dryhurst, S., Sahlin, U., Dufouil, C., Arwidson, P., Freeman, A. L. J., \& van der Linden, S. (2020). Predictors of COVID-19 vaccine acceptance across time and countries. MedRxiv. https://doi.org/10.1101/2020.12.09.20246439

Korn, L., Böhm, R., Meier, N. W., \& Betsch, C. (2020). Vaccination as a social contract. Proceedings of the National Academy of Sciences, 117(26), 14890-14899. https://doi.org/10.1073/pnas.1919666117

Lindholt, M. F., Jørgensen, F. J., Bor, A., \& Petersen, M. (2020, November 27). Willingness to Use an Approved COVID-19 Vaccine: Cross-National Evidence on Levels and Individual-Level Predictors. PsyArXiv. https://doi.org/10.31234/osf.io/8kn5f

Logan, J., Nederhoff, D., Koch, B., Griffith, B., Wolfson, J., Awan, F. A., \& Basta, N. E. (2018). 'What have you HEARD about the HERD?' Does education about local influenza vaccination coverage and herd immunity affect willingness to vaccinate? Vaccine, 36(28), 4118-4125. https://doi.org/10.1016/j.vaccine.2018.05.037

MacDonald, N. E., \& SAGE Working Group on Vaccine Hesitancy (2015). Vaccine hesitancy: Definition, scope and determinants. Vaccine, 33(34), 4161-4164. https://doi.org/10.1016/j.vaccine.2015.04.036

McAuliffe, W. H. B., Carter, E. C., Berhane, J., Snihur, A. C., \& McCullough, M. E. (2020). Is empathy the default response to suffering? A meta-analytic evaluation of perspective taking's effect on empathic concern. Personality and Social Psychology Review, 24(2), 141-162. https://doi.org/10.1177/1088868319887599 
Mullen O'Keefe, S. (2020). One in three Americans would not get COVID-19 vaccine. Gallup. https://news.gallup.com/poll/317018/one-threeamericans-not-covidvaccine.aspx

Murphy, J., Vallières, F., Bentall, R. P., Shevlin, M., McBride, O., Hartman, T. K., McKay, R., Bennett, K., Mason, L., Gibson-Miller, J., Levita, L., Martinez, A. P., Stocks, T. V. A., Karatzias, T., \& Hyland, P. (2020). Preparing for a COVID-19 vaccine: Identifying and psychologically profiling those who are vaccine hesitant or resistant in two general population samples. PsyArXiv. https://doi.org/10.31234/osf.io/pev2b

Pardi, N., Hogan, M. J., Porter, F. W., \& Weissman, D. (2018). mRNA vaccines - a new era in vaccinology. Nature reviews. Drug discovery, 17(4), 261-279. https://doi.org/10.1038/nrd.2017.243

Pfattheicher, S., Nockur, L., Böhm, R., Sassenrath, C., \& Petersen, M. B. (2020). The emotional path to action: Empathy promotes physical distancing and wearing of face masks during the COVID-19 pandemic. Psychological Science, 31(11), 1363-1373. https://doi.org/10.1177/0956797620964422

Pfattheicher, S., Sassenrath, C., \& Keller, J. (2019). Compassion magnifies third-party punishment. Journal of Personality and Social Psychology, 117(1), 124-141. https://doi.org/10.1037/pspi0000165

Promislow, D. E. L. (2020). A geroscience perspective on COVID-19 mortality. The Journals of Gerontology: Series A, 75(9), e30-e33. https://doi.org/10.1093/gerona/glaa094

Rosenberg, B. D., \& Siegel, J. T. (2018). A 50-year review of psychological reactance theory: Do not read this article. Motivation Science, 4(4), 281-300. https://doi.org/10.1037/mot0000091

Rubin, L. G., Levin, M. J., Ljungman, P., Davies, E. G., Avery, R., Tomblyn, M., Bousvaros, A., Dhanireddy, S., Sung, L., Keyserling, H., \& Kang, I. (2014). 2013 IDSA clinical practice guideline for vaccination of the immunocompromised host. Clinical Infectious Diseases, 58(3), e44-e100. https://doi.org/10.1093/cid/cit816 
Sanche, S., Lin, Y. T., Xu, C., Romero-Severson, E., Hengartner, N., \& Ke, R. (2020). High contagiousness and rapid spread of severe acute respiratory syndrome coronavirus 2 . Emerging Infectious Diseases, 26(7), 1470-1477. https://doi.org/10.3201/eid2607.200282

Sassenrath, C., Diefenbacher, S., Siegel, A., \& Keller, J. (2016). A person-oriented approach to hand hygiene behaviour: Emotional empathy fosters hand hygiene practice. Psychology \& Health, 31(2), 205-227. https://doi.org/10.1080/08870446.2015.1088945

Sherman, S. M., Smith, L. E., Sim, J., Amlôt, R., Cutts, M., Dasch, H., Rubin, G. J., \& Sevdalis, N. (2020). COVID-19 vaccination intention in the UK: results from the COVID-19 vaccination acceptability study (CoVAccS), a nationally representative cross-sectional survey. Human Vaccines \& Immunotherapeutics, 1-10. Advance online publication. https://doi.org/10.1080/21645515.2020.1846397

Shim, E., Chapman, G. B., Townsend, J. P., \& Galvani, A. P. (2012). The influence of altruism on influenza vaccination decisions. Journal of the Royal Society Interface, 9(74), 2234 2243. https://doi.org/10.1098/rsif.2012.0115

Siddique, H. (2020, november 10). Coronavirus: Anti-vaxxers seek to discredit Pfizer's vaccine. The Guardian. https://www.theguardian.com/world/2020/nov/10/coronavirus-antivaxxers-seek-to-discredit-pfizers-vaccine

Soto, C. J. (2019). How replicable are links between personality traits and consequential life outcomes? The life outcomes of personality replication project. Psychological Science, 30(5), 711-727. https://doi.org/10.1177/0956797619831612

Sprengholz, P., \& Betsch, C. (2020). Herd immunity communication counters detrimental effects of selective vaccination mandates: Experimental evidence. EClinicalMedicine, 22. https://doi.org/10.1016/j.eclinm.2020.100352

Sprengholz, P., Felgendreff, L., Böhm, R., \& Betsch, C. (2020). Detrimental effects of mandating vaccination against COVID-19 and how to counteract them [unpublished manuscript]. Friedrich Schiller University Jena. 
Thielmann, I., Spadaro, G., \& Balliet, D. (2020). Personality and prosocial behavior: A theoretical framework and meta-analysis. Psychological Bulletin, 146(1), 30-90. https://doi.org/10.1037/bul0000217

Thomson, A., Robinson, K., \& Vallée-Tourangeau, G. (2016). The 5As: A practical taxonomy for the determinants of vaccine uptake. Vaccine, 34(8), 1018-1024. https://doi.org/10.1016/j.vaccine.2015.11.065

Todd, A. R., \& Burgmer, P. (2013). Perspective taking and automatic intergroup evaluation change: Testing an associative self-anchoring account. Journal of Personality and Social Psychology, 104(5), 786-802. https://doi.org/10.1037/a0031999

WHO. (2020a). Coronavirus disease (COVID-19): Herd immunity, lockdowns and COVID-19. WHO. https://www.who.int/news-room/q-a-detail/herd-immunity-lockdowns-and-covid$\underline{19}$

WHO. (2020b). Draft landscape of COVID-19 candidate vaccines. WHO. https://www.who.int/publications/m/item/draft-landscape-of-covid-19-candidate-vaccines

WHO. (2020c). Managing the COVID-19 infodemic: Promoting healthy behaviours and mitigating the harm from misinformation and disinformation. WHO.

https:/www.who.int/news-room/detail/23-09-2020-managing-the-covid-19-infodemicpromoting-healthy-behaviours-and-mitigating-the-harm-from-misinformation-and$\underline{\text { disinformation }}$

WHO. (2020d). WHO Director-General's opening remarks at the media briefing on COVID-19 21 August 2020. WHO. https://www.who.int/dg/speeches/detail/who-director-general-sopening-remarks-at-the-media-briefing-on-covid-19---21-august-2020 